\title{
Características físicas de ovos de galinhas nativas comparadas a linhagem de postura
}

Almeida, E.C.J. ${ }^{1 @ ;}$ Carneiro, P.L.S. ${ }^{2}$; Nunes, L.A. ${ }^{3}$; Pereira, A.H.R. ${ }^{4}$; Farias Filho, R.V. ${ }^{5}$; Malhado, C.H.M. ${ }^{2}$ e Bittencourt, T.C.B.S.C. ${ }^{1}$

'Escola de Medicina Veterinária e Zootecnia. Universidade Federal da Bahia. Salvador, BA. Brasil.

${ }^{2}$ Departamento de Ciências Biológicas. Universidade Estadual do Sudoeste da Bahia. Jequié, BA. Brasil.

${ }^{3}$ Programa de Pós-graduação em Enfermagem e Saúde, Universidade Estadual do Sudoeste da Bahia, Jequié, BA. Brasil.

4Programa de Pós-graduação em Zootecnia, Universidade Estadual Vale do Acaraú, Sobral, CE. Brasil.

${ }^{5}$ Departamento de Ciência Rural e Animal. Universidade Estadual do Sudoeste da Bahia. Itapetinga, BA. Brasil.

\section{PALAVRAS CHAVE ADICIONAIS}

Avicultura caipira.

Conservação.

Diversidade fenotípica.

Multivariada.

Poedeiras.

\section{RESUMO}

Objelivou-se avaliar a variação nas características físicas dos ovos entre raças de galinhas nativas e uma linhagem comercial de postura. Foram mesuradas as características de peso do ovo, comprimento do ovo, largura do ovo, diâmetro da gema, peso da gema, peso do albúmen, espessura da casca, peso da casca e índice da forma. Utilizou-se um total de 528 ovos das raças Peloco, Barbuda e Caneluda e da linhagem comercial de ovos brancos Lohmann. Foi realizada a avaliação da contribuição relativa das variáveis e, posteriormente, os dados foram submetidos às análises de componentes principais e agrupamento. O primeiro componente principal foi caracterizado como um índice do tamanho do ovo que explica $77,0 \%$ da variação nos ovos. Houve a formação de dois grupos sendo um formado pela raça Caneluda e a linhagem comercial e o outro pelas raças Barbuda e Peloco. A raça Caneluda produz ovos com características semelhantes à linhagem comercial e maiores que as raças Barbuda e Peloco. Os ovos das galinhas nativas podem ser categorizados de médios a extragrande de acordo com os pesos.

\section{Physical characteristics of eggs of native chickens compared to laying lineage}

\begin{abstract}
SUMMARY
The aim of this study was to evaluate the variation on the physical traits of the eggs between the breed native chicken and a commercial egg layer lineage. Were measured the traits: egg weight, egg length, egg width, yolk diameter, yolk weight, albumen weight, thickness shell, shell weight and shape index. We used a total of 528 eggs from Peloco, Barbuda and Caneluda breeds and of a commercial whiteegg Lohmann lineage. The relative contribution of the variables was evaluated and, subsequently, the data were summited to principal components and cluster analysis. The first principal component explained $77.0 \%$ of the variation among the eggs and it was identified as index of the egg size. There was the formation of two groups, one of which with the Caneluda breed and the commercial lineage and the other one with Barbuda and Peloco breeds. Caneluda breed produces eggs with some features alike the commercial lineage and bigger than the ones of the Barbuda and Peloco breeds. It was possible to characterize the eggs of native chicken from medium to extra-large, according to their weight.
\end{abstract}

\section{INTRODUÇÃO}

A produção industrial de ovos no Brasil é de aproximadamente 37 bilhões de ovos por ano, dos quais $99 \%$ são destinados ao consumo interno que é de 182 ovos/ habitante/ano (ABPA, 2016). O ovo é um alimento essencial na composição da dieta humana e considerado uma proteína de alto valor biológico. Além disso, é um alimento de baixo custo e acessível para o consumidor de menor poder aquisitivo, sendo que as características físicas e químicas do ovo podem influenciar o seu grau de aceitabilidade no mercado e também agregar valor ao produto comercializado (Pascoal et al., 2008; Freitas et al., 2011).

Embora a produção nacional de ovos em grande escala seja exclusivamente em sistema industrial, o sistema de produção de ovos do tipo caipira é um tipo de criação alternativo que tem se destacado. Isto, principalmente, pela tendência dos consumidores op- 
tarem por produtos oriundos de sistemas de criação em acordo com as regras de segurança alimentar e bem-estar animal, além das características diferenciadas associadas à qualidade e sabor dos ovos caipira. Em muitos casos, os sistemas de produção caipira são fundamentados na agricultura familiar que é baseada nos princípios da sustentabilidade econômica, social e ambiental dos sistemas de produção (Reichert et al., 2011).

As galinhas nativas possuem características essenciais para sobrevivência em sistemas com poucos recursos e menor disponibilidade de alimentos, o que as torna adaptadas aos sistemas de criação caipira (Mengesha e Tsega, 2011). Especialmente nos países em desenvolvimento, podem contribuir para a diversificação do setor avícola, objetivando obtenção de recursos genéticos adaptados as condições locais. Além disso, são importante fonte de renda para o pequeno produtor (Yakubo et al., 2008; Aldersm e Pym, 2010, Alderson, 2018).

As raças de galinhas nativas brasileiras encontramse em estado eminente de desaparecimento ou em pequenos núcleos subutilizados em criações caseiras. Este cenário deve-se em grande parte a substituição massiva destas raças pelas linhagens melhoradas geneticamente e altamente produtivas, que ocorreu durante o processo de expansão da avicultura industrial no país. De acordo com Moula et al. (2010), estratégias voltadas para a caracterização e conservação destes recursos genéticos são necessárias. Dentre estas, ressalta-se a avaliação das características produtivas e de importância econômica.

No Núcleo de Conservação de Aves Nativas, da Universidade Estadual do Sudoeste da Bahia são mantidos alguns grupos raciais de galinhas nativas entre estas as raças Peloco, Barbuda e Caneluda (Figura 1), contudo, informações populacionais, fenotípicas, genéticas e produtivas dessas galinhas são desconhecidas e suas potencialidades precisam ser avaliadas. Assim, objetivou-se avaliar a diversidade nas características físicas do ovo entre três raças de galinhas nativas e uma linhagem comercial de postura.

\section{MATERIAL E METODOS}

Foram utilizadas 135 fêmeas das raças de galinhas nativas Peloco $(n=51)$, Barbuda $(n=12)$ e Caneluda $(n=22)$ e a linhagem comercial Lohmann (50). As galinhas foram separadas em lotes conforme o grupo genético para a coleta dos ovos por grupo. As galinhas nativas foram provenientes do Núcleo de Conservação de Aves Nativas do Laboratório Experimental de Avicultura da Universidade Estadual do Sudoeste da Bahia (UESB), localizado na cidade de Itapetinga, Bahia.

Utilizou-se 528 ovos das raças nativas Peloco (158), Barbuda (87) e Caneluda (63) e da linhagem comercial Lohmann (220). A variação no total de ovos/grupo foi influenciada pelo número de aves disponíveis e a taxa de postura diária de cada raça, contudo, as raças Barbuda e Caneluda possuem menor tamanho populacional pelo fato de estarem em fase inicial de conservação.

A linhagem comercial de postura foi adquirida de uma granja localizada na cidade de Vitória da Conquista, Bahia. As galinhas foram criadas soltas no piso em galpão semiaberto recebendo dieta apropriada às exigências nutricionais de poedeiras. As dietas utilizadas foram formuladas à base de milho e farelo de soja para atender a exigência de $2800 \mathrm{kcal} / \mathrm{kg}$ de energia metabolizável, $15 \%$ de proteína bruta, 4\% de cálcio e $0,3 \%$ de fósforo (Rostagno et al., 2011), seguindo a seguinte composição dos ingredientes $(\mathrm{kg} / 100 \mathrm{~kg}$ de ração): milho $71,47 \mathrm{~kg}$; farelo de soja $17 \mathrm{~kg}$; fosfato bicálcico 1,41kg; calcário calcítico $8,77 \mathrm{~kg}$; mistura vitamínica e mineral $0,5 \mathrm{~kg}$; óleo de soja, $0,5 \mathrm{~kg}$. Foram utilizadas galinhas adultas, no terço final de postura (65 a $70^{\mathrm{a}}$ semana).

Os ovos foram coletados quatro vezes ao dia durante cinco semanas de fevereiro a março de 2015, sendo higienizados e identificados para posterior avaliação. Com os ovos ainda íntegros foi determinado o peso do ovo, o comprimento do ovo e a largura do ovo. Em seguida, os ovos foram cuidadosamente quebrados sobre uma mesa de vidro para a medição do diâmetro da gema. A gema e

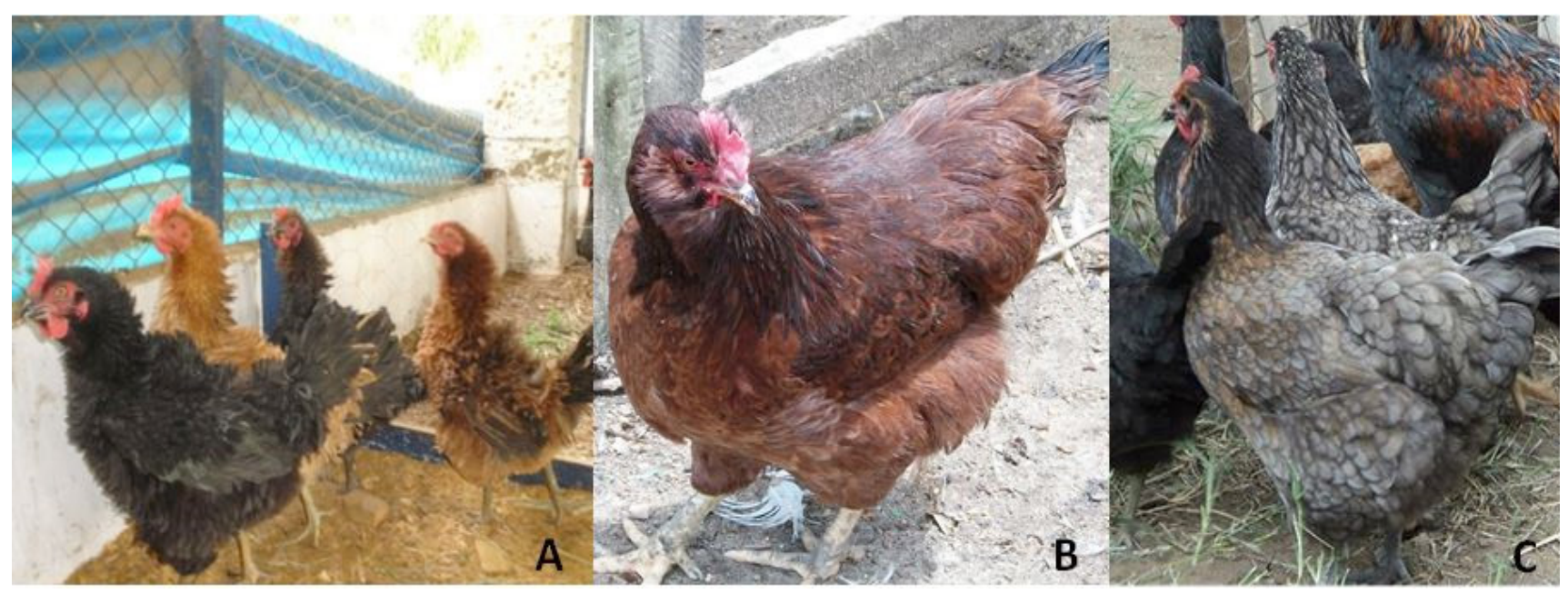

Figura 1. Fêmeas de galinhas das raças nativas Peloco (A), Barbuda (B) e Caneluda (C) (Females chickens of the native breeds Peloco (A), Barbuda (B) and Caneluda (C)). 
o albumen foram então separados manualmente para obtenção das medidas de peso da gema e peso do albúmen. O peso do albumen foi obtido pela diferença entre o peso total, peso da gema e peso da casca. Após a secagem das cascas ao ambiente por $48 \mathrm{~h}$, sem remoção da membrana interna, foi obtido o peso da casca. Foram tomadas oito medidas em pontos diferentes da casca, sendo quatro em cada metade, para obtenção da espessura da casca. A partir da relação entre largura e comprimento do ovo foi obtido o índice da forma do ovo (\%). As medidas de peso do ovo, peso da gema e peso da casca foram realizadas com uso de balança eletrônica (precisão 0,001 kg). Enquanto comprimento do ovo, largura do ovo, diâmetro da gema e espessura da casca foram coletadas utilizando o paquímetro digital (precisão 0,01 mm).

Os dados foram submetidos à análise de variância (ANOVA), para avaliar o efeito do grupo genético. As médias foram comparadas pelo teste de Tukey a 5\% de probabilidade. Utilizou-se o modelo estatístico:

$$
\mathrm{Y}_{\mathrm{itr}}=\mu_{\mathrm{t}}+\mathrm{G}_{\mathrm{it}}+\mathrm{e}_{\mathrm{itr}}
$$

em que: $\mathrm{Y}_{\text {itr }}$ é o valor da característica $t$, no grupo genético i, no animal $r$; $\mu_{\mathrm{t}}$ é a média da característica $t$; $\mathrm{G}_{\mathrm{it}}$ é o efeito do grupo genético i na característica t e $\mathrm{e}_{\mathrm{itr}}$ é o efeito do erro aleatório atribuído à observação $Y_{i t r}$.

Foi realizada uma análise de variância multivariada (MANOVA), para avaliação do efeito de grupo genético sobre as variáveis simultaneamente, conforme o modelo descrito abaixo:

$$
\mathrm{y}=\mathrm{X} \beta+\varepsilon,
$$

em que: y é o vetor de observações para cada variável; $\beta$ é o vetor de efeitos fixos para tratamento; $X$ é a matriz de incidência dos efeitos fixos e $\varepsilon$ é o vetor de erros aleatórios associados a cada observação.

Em seguida foi realizada seleção de variáveis, via avaliação da contribuição relativa das variáveis para a variação entre os grupos pelo critério de Singh (1981), que resultou no descarte de largura do ovo $(1,1 \%)$, diâmetro da gema $(0,8 \%)$ e espessura da casca $(0,1 \%)$, que apresentaram menor importância relativa. As variáveis de menor contribuição geralmente são invariantes ou correlacionadas, portanto, passíveis de descarte (Meira et al., 2013).

Após o descarte, o novo conjunto de variáveis formado por peso do ovo, comprimento do ovo, peso do albúmen, peso da gema, peso da casca e índice da forma foi padronizado e submetido a uma análise de componentes principais, possibilitando a obtenção do gráfico biplot para avaliação da relação entre as variáveis e os grupos raciais.

Posteriormente, foi realizada uma análise de agrupamento pelo método hierárquico UPGMA (Unweighted Pair Group Method With Arithmetic Mean), adotando a distância Euclidiana como medida de dissimilaridade. A estabilidade dos agrupamentos foi testada pela análise de Bootstrap, com 1.000 repetições.

Para a ANOVA e MANOVA foi utilizado o software estatístico SAS (2004). A análise da contribuição relativa das variáveis foi executada no programa GENES (Cruz, 2009) e as análises de componentes principais e agrupamento foram feitas no programa PAST versão 2.03 (Hammer et al., 2001).

\section{RESULTADOS}

Foram verificadas diferenças significativas $(p<0,05)$ para todas as características, exceto espessura da casca. A linhagem comercial apresentou as maiores médias para comprimento do ovo e peso do albúmen, já os ovos da Caneluda apresentaram maior peso e diâmetro da gema (Tabela I). Os grupos com as maiores médias para peso da gema, albúmen e casca tiveram maior percentual desses componentes no ovo e consequentemente maior peso do ovo. A raça Caneluda e a linhagem comercial não diferiram quanto ao peso e largura do ovo, porém, apresentaram diferenças significativas para estas características em relação à Barbuda e Peloco (Tabela I).

As raças nativas não diferiram entre si quanto ao índice de forma, porém, apresentaram índices supe-

\begin{tabular}{|c|c|c|c|c|c|}
\hline Características & Comercial & Caneluda & Barbuda & Peloco & Prob. \\
\hline Peso do ovo (g) & $66,21^{a}$ & $64,65^{\mathrm{a}}$ & $56,01^{b}$ & $52,50^{c}$ & $<0,0001$ \\
\hline Comprimento do ovo (mm) & $60,09^{a}$ & $58,13^{b}$ & $55,20^{c}$ & $54,08^{d}$ & $<0,0001$ \\
\hline Largura do ovo (mm) & $44,42^{a}$ & $44,29^{a}$ & $42,44^{b}$ & $41,19^{c}$ & $<0,0001$ \\
\hline Diâmetro da gema (mm) & $42,15^{\mathrm{b}}$ & $42,96^{a}$ & $38,36^{c}$ & $38,89^{c}$ & 0,0938 \\
\hline Peso do albúmen (g) & $43,81^{a}$ & $40,51^{b}$ & $37,56^{c}$ & $32,95^{d}$ & $<0,0001$ \\
\hline Peso da gema (g) & $17,27^{\mathrm{b}}$ & $18,14^{a}$ & $13,74^{d}$ & $14,65^{\mathrm{c}}$ & $<0,0001$ \\
\hline Peso da casca $(\mathrm{g})$ & $5,13^{b}$ & $6,00^{\mathrm{a}}$ & $4,70^{c}$ & $4,89^{\mathrm{bc}}$ & $<0,0001$ \\
\hline Espessura da casca $(\mathrm{mm})$ & $0,36^{a}$ & $0,37^{a}$ & $0,35^{a}$ & $0,38^{a}$ & 0,3297 \\
\hline Índice de Forma (\%) & $74,06^{b}$ & $76,30^{\mathrm{a}}$ & $76,98^{a}$ & $76,24^{a}$ & $<0,0001$ \\
\hline
\end{tabular}
riores a linhagem comercial (Tabela I). Já com relação

$\mathrm{g}$ = gramas; $\mathrm{mm}$ = milimétros; Prob. = probabilidade.

Médias com letras diferentes na linha difererem entre si pelo teste de Tukey, ao nível de $5 \%$ de probabilidade $(P<0,05)$. 


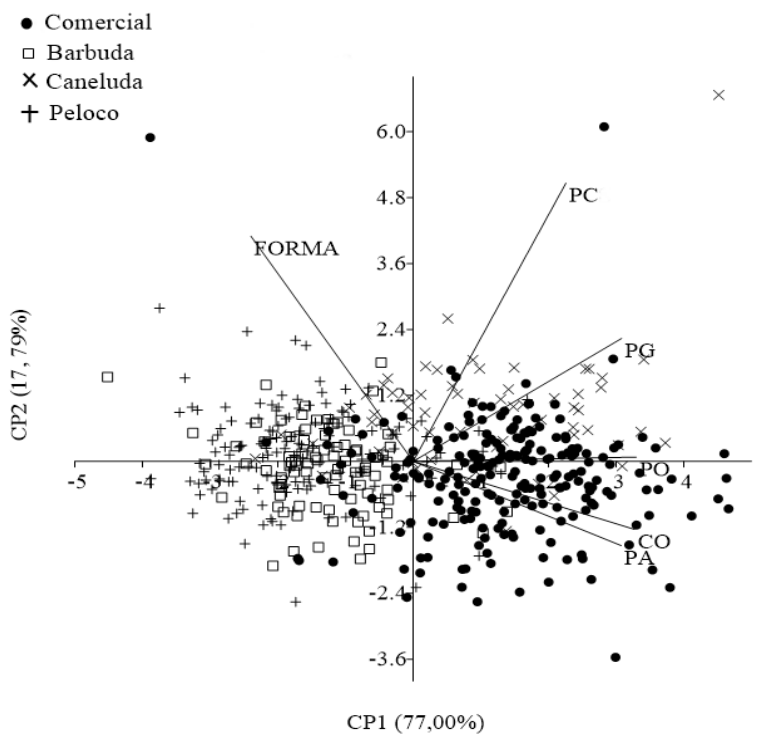

Figura 2. Biplot baseado nos dois primeiros componentes principais (CP1 e CP2) obtidos das medidas dos ovos das raças de galinha Barbuda, Caneluda e Peloco e uma linhagem comercial. $\mathrm{PO}=$ peso do ovo; $\mathrm{CO}=$ comprimento do ovo; $\mathrm{PA}=$ peso do albúmen; $\mathrm{PG}$ = peso da gema; $\mathrm{PC}=$ peso da casca; FORMA = índice de forma (Biplot based on the first two principal components (CP1 and CP2) obtained from the measurements of the eggs of Barbuda, Caneluda and Peloco chicken breeds and a commercial lineage. PO = egg weight; $\mathrm{CO}=$ egg length; $\mathrm{PA}=$ albumen weight; $\mathrm{PG}=$ weight yolk; PC = shell weight; FORMA = shape index).

ao peso da casca foi verificada maior média para ovos da raça Caneluda, enquanto as raças Barbuda e Peloco não diferiram entre si. No entanto, os ovos das galinhas Peloco tiveram peso da casca igual à linhagem comercial (Tabela I).

Foram verificadas diferenças significativas $(p<0,0001)$ entre os grupos genéticos pelo teste de Wilks da análise de variância multivariada (MANOVA). As variáveis de maior contribuição para a variação entre os grupos genéticos foram peso do albúmen $(31,6 \%)$, peso da gema $(20,7 \%)$, peso do ovo $(20,0 \%)$, comprimento do ovo $(13,0 \%)$, peso da casca $(9,4 \%)$ e índice de forma $(4,0 \%)$. Pode-se afirmar que as características de maior contribuição são as que apresentaram maior variação entre os grupos genéticos.

Os dois primeiros componentes principais explicaram $94,8 \%$ da variação entre os grupos raciais. Verificou-se, pelo direcionamento de seus vetores que as variáveis peso do ovo, comprimento do ovo, peso do albúmen e peso da gema estão fortemente associadas (r $>0,90)$ com o CP1 (Figura 2), que explicou 77,0\% da variação entre os grupos e foi descrito como: CP1 $=0,4600^{*}$ peso do ovo $+0,4564^{*}$ comprimento do ovo $+0,4289^{*}$ peso do albúmen $+0,4300^{*}$ peso da gema + $0,3149^{*}$ peso da casca $-0,3347^{*}$ forma.

Verificou-se ainda maior proximidade entre a linhagem comercial e a raça Caneluda, que ficaram dispostos na mesma direção dos vetores das características peso do ovo, peso do albúmen, peso da gema e peso da casca (Figura 2). Este resultado evidencia o desempenho superior de ambos os grupos para estas variáveis.
A análise de agrupamento comprovou os resultados visualizados na dispersão gráfica, resultando na formação de dois grupos, sendo um formado pelas raças Barbuda e Peloco e o outro pela raça Caneluda e a linhagem comercial, com bootstrap de 100\% (Figura 3).

\section{DISCUSSÃO}

O peso do ovo reflete o aumento na quantidade de gema, albúmen e casca no ovo, sendo que aproximadamente $65 \%$ do peso é constituído pelo albúmen, 25\% pela gema e 10\% pela casca (Santos et al., 2009). É uma característica extremamente importante na produção de ovos, relacionada com o processo de incubação e com o peso do pintinho ao nascimento (Lourens et al., 2006; Rocha et al., 2008).

A variação na produção e peso dos ovos pode ser influenciada por diversos fatores como nutrição, genética, idade da ave e período de postura (Bouvarel e Nys, 2011). Estudos recentes têm utilizado novas abordagens, como redes neurais, para avaliar os efeitos desses fatores na curva de produção de ovos de galinhas (Galeano-Vasco et al., 2018). O maior peso do ovo verificado para as galinhas da raça Caneluda, entre as raças nativas, pode ser justificado por seu maior porte. Sabe-se que aves mais pesadas tendem a produzir ovos mais pesados. Acredita-se que esta raça tenha em sua formação participação de raças americanas, que são aves pesadas e que produzem ovos grandes.

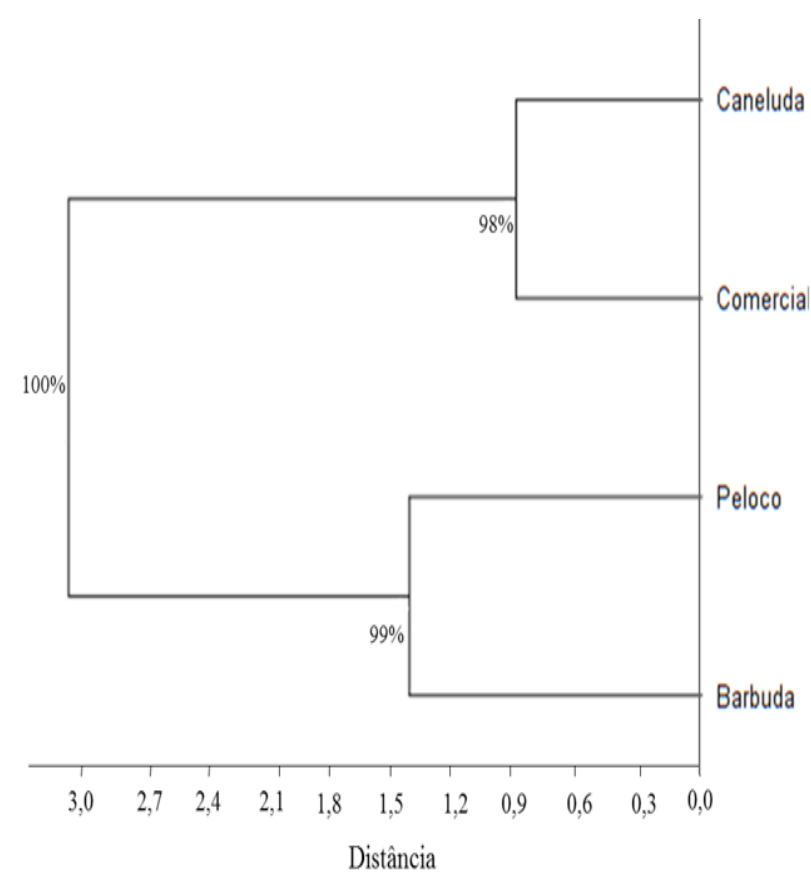

Figura 3. Dendrograma obtido a partir da distância de Mahalanobis para as raças de galinhas nativas Barbuda, Caneluda e Peloco e uma linhagem comercial de postura pelo método de agrupamento UPGMA (Unweighted pair group method with arithmetic mean), com 1000 repetições de bootstrap. (Dendrogram obtained from the Mahalanobis distance for the Barbuda, Caneluda and Peloco breeds native chickens and a commercial laying lineage by UPGMA (Unweighted pair group method with arithmetic mean), with 1000 replicates of bootstrap). 
O peso do ovo é um dos fatores determinante para o preço do produto final nos supermercados. De acordo com o Ministério da Agricultura, Pecuária e Abastecimento (MAPA, 1991), os ovos são classificados pelo tamanho em quatro tipos: tipo1, extragrande (peso mínimo de 60g); tipo 2, grande (peso mínimo de 55g); tipo 3, médio (peso mínimo de $50 \mathrm{~g}$ ) e tipo 4, pequeno (peso mínimo de 45g). As raças Peloco, Barbuda e Caneluda apresentaram ovos com pesos médios de aproximadamente $52 \mathrm{~g}, 56 \mathrm{~g}$ e $64 \mathrm{~g}$, respectivamente (Tabela I), podendo ser classificados como médio, grande e extragrande. Estes valores foram superiores aos encontrados em outros estudos, que evidenciaram o menor tamanho do ovo de galinhas locais (menores que 50g), categorizando-os como ovos pequenos (Parmar et al., 2006; Yakubu et al., 2008; Iqbal et al., 2012).

O peso da casca está relacionado com o percentual de casca no ovo, e junto com a espessura da casca constituem dois indicadores de resistência do ovo a danos físicos. Segundo Pelícia et al. (2007) a resistência da casca permite ao ovo resistir aos impactos da postura, colheita e classificação, na granja, até o transporte, armazenamento e comercialização dos ovos. Assim, é uma característica importante, especialmente no sistema de criação caipira, em que os ovos são mais expostos, sendo necessária em todas as fases da produção até o consumidor final.

Pode-se observar que embora as aves deste estudo estivessem entre 65 e 70 semanas de idade, podendo ser consideradas aves velhas, os valores de espessura da casca do ovo foram para todos os grupos superiores a $0,33 \mathrm{~mm}$. Em galinhas, ovos com espessura de casa inferior a $0,33 \mathrm{~mm}$ apresentam maiores chances de sofrerem danos físicos (Stadelman e Cotterill, 1995).

Com relação a análise multivariada, nota-se que os escores do primeiro componente principal (CP1) foram positivos e próximos, exceto pra o índice de forma que obteve escore negativo. Portanto, o CP1 representa um contraste entre as demais variáveis e o índice de forma, ou seja, se o comprimento do ovo e o peso do ovo e seus componentes (albúmen, gema e casca) forem baixos e o índice de forma for alto, menor será o primeiro componente. Assim, o CP1 pode ser rotulado como uma diferença no tamanho dos ovos, representado pelo seu peso e comprimento e o formato dos ovos, representado pelo índice da forma, sendo que mais de $70,0 \%$ da variação entre os grupos genéticos decorrem das variações nessa diferença.

A raça Caneluda produziu ovos com características externas e internas mais próximas a linhagem comercial, embora tratar-se de aves com porte corporal maior, diferente do padrão fenotípico das linhagens comerciais de ovos brancos. Este grupo também se diferencia das galinhas Barbuda e Peloco, que são aves menores, assim, sua criação pode ser direcionada para a produção com duplo propósito, em que as fêmeas são utilizadas na produção de ovos e os machos voltados para o abate, em função do maior porte.

Geralmente, em sistemas de produção caipira a criação de duplo propósito é vantajosa como forma de maior aproveitamento dos animais no sistema. As raças Barbuda e Peloco são aves com padrão fenotípico mais próximo e que produzem ovos com características semelhantes, justificando sua maior proximidade na análise de agrupamento.

As poedeiras comerciais são geralmente aves leves e mais eficientes por serem selecionadas para produção exclusiva de ovos em maior quantidade e qualidade de comercialização, com menor conversão alimentar. No entanto, Moula et al. (2010), verificaram que galinhas locais apresentaram maior resistência da casca do ovo quando comparadas a linhagens comerciais. Segundo estes autores, considerando que as galinhas locais não foram selecionadas para as características de produção de ovos, tal desempenho pode ser considerado satisfatório, além disso, práticas de melhoramento das características dos ovos devem ser consideradas para as raças locais.

Neste sentido, os ovos das galinhas nativas podem apresentar maior valor agregado, relacionado com o modo de criação caipira, que lhes confere sabor diferenciado e, também a variedade na coloração da casca dos ovos, atributo apreciado por alguns consumidores. Além disso, a importância econômica e sociocultural que estas aves possuem para a avicultura familiar sustentável que deve ser considerada, podendo resultar em maior rentabilidade para o pequeno produtor.

\section{CONCLUSÃO}

A diferença entre tamanho e forma do ovo das galinhas é o fator que mais diferencia os grupos genéticos, sendo que o ecótipo Caneluda e a linhagem comercial são mais semelhantes, assim como os ecótipo Barbuda e Peloco. Os ovos dos ecótipos Peloco, Barbuda e Caneluda podem ser categorizados como médio, grande e extragrande agregando maior valor comercial.

\section{AGRADECIMENTOS}

À Fundação de Amparo à Pesquisa do Estado da Bahia (FAPESB) pela concessão da bolsa de estudos e apoio financeiro recebido e à Universidade Estadual do Sudoeste da Bahia (UESB) pela concessão dos animais experimentais.

\section{BIBLIOGRAFIA}

Alders, RG \& Pym, RAE 2010, 'Village poultry: still important to millions, eight thousand year after domestication', World's Poultry Science Journal, vol.65, no. 2, pp. 181-190. doi.org/10.1017/ S0043933909000117.

Alderson, GLH 2018, 'Conservation of breeds and maintenance of biodiversity: justification and methodology for the conservation of Animal Genetic Resources', Archivos de Zootecnia, vol.67, no. 258, pp. 300-309.

Associação Brasileira de Proteína Animal (ABPA) 2015, Relatório Anual, acesso em 01 de janeiro de 2016,<http://abpa-br.com.br/files/ RelatorioAnual_UBABEF_2015_DIGITAL.pdfs.

Bouvarel, JR \&Nys, Y 2011, 'Hen nutrition for sustained egg quality', in: Nys, Y, Bain, M \& Van Immerseel, F (eds.), Improving the safety and quality of eggs and egg products, Wood Head Publishing, Cambridge, pp. 261-299.

Cruz, CD 2009, Programa Genes: Biometria, Editora UFV, Viçosa.

Freitas, LW, Paz, I, Garcia, MG, Caldara, F, Seno, L, Felix, G, Lima, H, Ferreira, M \& Cavichiolo, F 2011, 'Aspectos qualitativos de ovos 
comerciais submetidos a diferentes condições de armazenamento', Revista Agrarian, vol. 4, no. 11, pp. 66-72.

Galeano-Vasco, L, Galvan, IM, Aler, R. \& Cerón-Muñoz, MF 2018, 'Forecasting egg production curve with neural networks', Archivos de Zootecnia, vol. 67, no. 257, pp. 81-86.

Hammer, O, Harper, DAT\& Ryan, PD 2001,'PAST: Paleontological Statistics of twere package for education and data analysis', Paleontologia Eletrônica, vol. 4, no. 1, pp. 1-9.

Iqbal, A, Akram, M, Sahota, AW, Javed, K, Hussain, J, Sarfraz, Z \& Mehmood, S 2012,'Laying characteristics and egg geometry of four varieties of Indigenous Aseel chicken in Pakistan', The Journal of Animal \& Plant Sciences, vol. 22, no. 4, pp. 848-852.

Lourens, A, Molenaar, R, Van Den Brand, H, Heetkamp, MJ, Meijerhof, $\mathrm{R} \&$ Kemp, B 2006,'Effect of egg size on heat production and the transition of energy from egg to hatchling', Poultry Science, vol. 85, no. 4, pp. 770-776.

Meira, CT, Pereira, IG, Farah, MM, Pires, AV, Garcia, DA \& Cruz, VAR 2013,'Seleção de características morfofuncionais de cavalos da raça Mangalarga Marchador por meio da análise de componentes principais', Arquivo Brasileiro de Medicina Veterinária e Zootecnia, vol. 65, no. 6, pp. 1843-1848. doi.org/10.1590/S010209352013000600036 .

Mengesha, M\&Tsega, W2011, 'Phenotypic and genotypic characteristics of indigenous chickens in Ethiopia: A review', African Journal of Agricultural Research, vol. 6, no. 24, pp. 5398-5404.

Ministério da Agricultura Pecuária e Abastecimento (MAPA) 1991, Padrão de identidade e qualidade para ovo integral: Resolução $N^{\circ} 005$ de 05 de julho de 1991, acesso em 04 de Novembro 2015, <http:// www.iberpharm.com.br/arquivos/RES05-05-07-1991.pdfs.

Moula, N, Antoine-Moussiaux, N, Decuypere, E, Farnir, F, Mertens, K, Baerdemaeker, J \& Leroy, P 2010,'Comparative study of egg quality traits in two Belgian local breeds and two commercial lines of chickens', Archiv Fur Geflugelkunde, vol. 74, no. 3, pp. 164-171.

Parmar, SNS, Thakur, MS,Tomar, SS \& Pilla, PVA 2006,'Evaluation of egg quality traits in indigenous Kadaknath breed of poultry', Livestock Research for Rural Development, vol. 18, no. 9, pp. 1-6.

Pascoal, LAF, Bento Jr, FA, Santos, WS, Silva, RS, Dourado, LRB \& Bezerra, APA 2008,'Qualidade de ovos comercializados em diferentes estabelecimentos na cidade de Imperatriz- $M A^{\prime}$, Revista Brasileira de Saúde Produção Animal, vol. 9, no. 1, pp. 150-157.

Pelícia, K.; Garcia, E.A.; Scherer, M.R.S.; Móri, C.; Dalanezi, J.A.; Faitarone, A.B.G.; Molino, A.B. \& Berto, D.A 2007. Alternative calcium source effects on commercial egg production and quality.
Brazilian Journal of Poultry Science, vol. 9, no. 2, pp. 105-109. doi. org/10.1590/S1516-635X2007000200005.

Reichert, L, Gomes, MV \& Schwengber, JE 2011, 'Avaliação técnica e econômica de um agroecossistema familiar de base ecológica na região Sul do Rio Grande do Sul', Revista Brasileira de Agrociência, vol. 17, no. 1, pp. 123-132.

Rocha, JSR, Lara, LC, Baião, NC,Cançado, SV, Baião, LEC\& Silva, TR 2008,'Efeito da classificação dos ovos sobre o rendimento de incubação e os pesos do pinto e do saco vitelino', Arquivo Brasileiro de Medicina Veterinária e Zootecnia, vol. 60, no. 4, pp. 979-986.

Rostagno, HS 2011 , Tabelas Brasileiras para aves e suínos: composição de alimentos e exigências nutricionais, $3^{\mathrm{a}}$ ed, Editora UFV, Viçosa.

Santos, JRG, Fornari, CM\& Téo, MA 2007, 'Influência da qualidade da casca do ovo sobre índices de produtividade de um incubatório industrial', Ciência Rural, vol. 37, no. 2, pp. 524-527.doi.org/10.1590/ S0103-84782007000200035.

Santos, MSV, Espíndola, GB, Lôbo, RNB, Fuentes, MFF, Carvalho, LE. \& Santos, ABE 2009, 'Desempenho e qualidade dos ovos de poedeiras comerciais submetidas às dietas com diferentes óleos vegetais', Revista Brasileira de Saúde e Produção Animal, vol. 10, no. 3, pp. 654-667.

SAS Institute Inc 2004, Statistical Analysis System user's guide, Version 9.0 ed, SAS Institute, Cary, USA.

Schmidt, GS, Figueiredo, EAP \& Ávila, VS 2002, Fatores que afetam a qualidade do pinto de corte. Artigos Embrapa suínos e aves, acesso em 01 de fevereiro de 2015, <http://www.cnpsa.embrapa.br>.

Stadelman, WJ \& Cotterill, P 1995, Egg science and technology, $4^{\text {a }}$ ed, Haworth, New York.

Teixeira, BB, Euclydes, RF, Teixeira, RB, Silva, LP, Torres, RA, Silva, FG,Lehner, HG. \& Caetano, GC 2013, 'Herdabilidade de características de produção e postura em matrizes de codornas de corte', Ciência Rural, vol. 43, no. 2, pp. 361-365.doi.org/10.1590/ S0103-84782013000200028.

Yakubu, A, Ogah, DM \& Barde, E 2008, 'Productivity and Egg Quality of Free Range Naked Neck and Normal Feathered Nigerian Indigenous Chicken', International Journal Poultry Science, vol. 7, no. 6, pp. 579-585.

Zeidler, G 2002, 'Shell egg quality and preservation', in Bell, DD \& Weaver, WD (eds.), Commercial chicken meat and egg production, Springer, North America,USA., p. 1199-1217. 